\title{
Acute Corneal Hydrops in a Young Patient with Down Syndrome
}

\author{
Mina Abdelmseih ${ }^{1 *}$ and Parikshit Dhir ${ }^{2}$ \\ ${ }^{1}$ Department of Ophthalmology, Augenklinik, Germany \\ ${ }^{2}$ Department of Ophthalmology, Dhir Hospital, Bhiwani, Haryana, India
}

*Corresponding author: Mina Abdelmseih, Department of Ophthalmology, Augenklinik, Germany

\begin{abstract}
A case of severe acute corneal hydrops with keratoconus associated with Down syndrome described in a 16-year-old male patient presented with a sudden unilateral painful visual loss in his left eye. The anterior segment of his left eye showed a severe diffuse edematous ectasia of acute corneal hydrops. Eye rubbing of his left eye was observed. Continued gradual improvement in his visual acuity and a central corneal scar were detected.
\end{abstract}

\section{Introduction}

Down's syndrome is frequently associated with keratoconus, a chronic non-inflammatory disorder which is characterized by progressive thinning of the cornea leading to scarring and progressive stromal thinning. Its incidence in Down's syndrome

has been reported up to $15 \%$ [1]. Acute corneal hydrops is characterized by marked stromal edema due to a tear in Descemet membrane (DM) which permits aqueous to enter the corneal stroma and epithelium [2-3].

\section{Case Report}

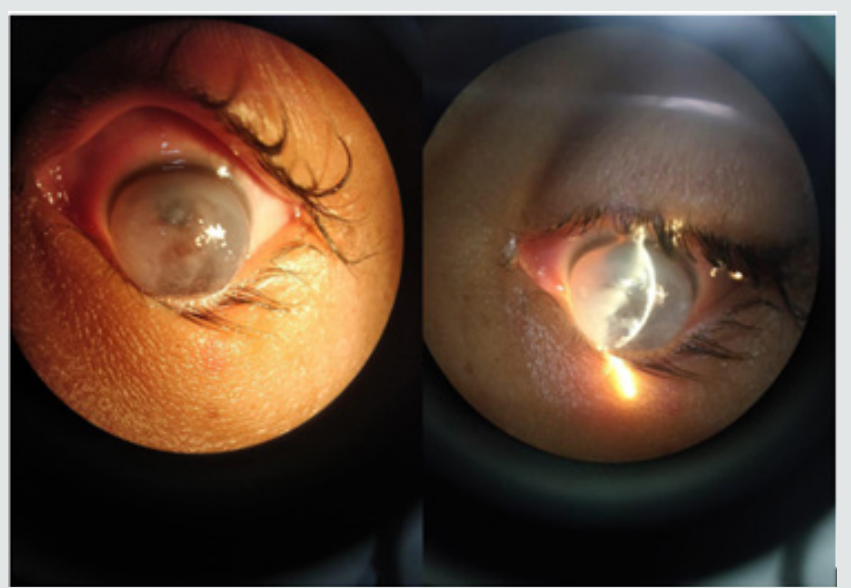

Figure 1: Color photography of the left eye showed severe microcystic edema, bullae and conical protrusion. 


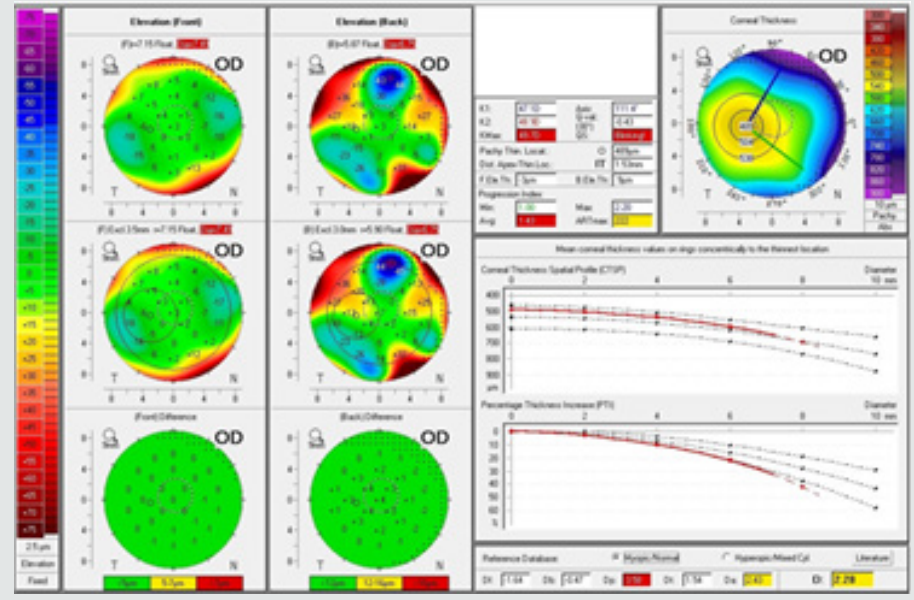

Figure 2: Pentacam of the right eye showed an early stage of Keratoconus.

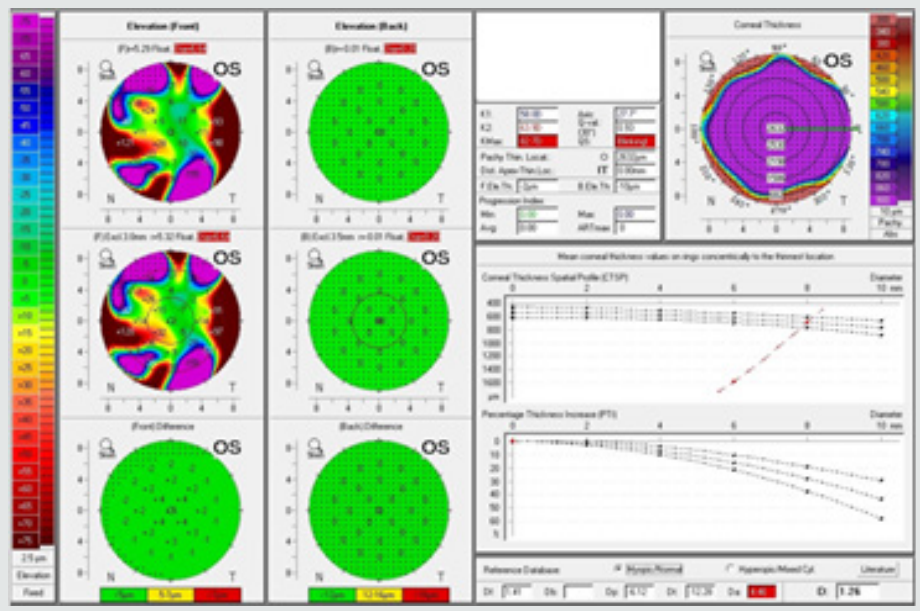

Figure 3: Pentacam of the left eye revealed marked increase in the corneal thickness because of severe edema.

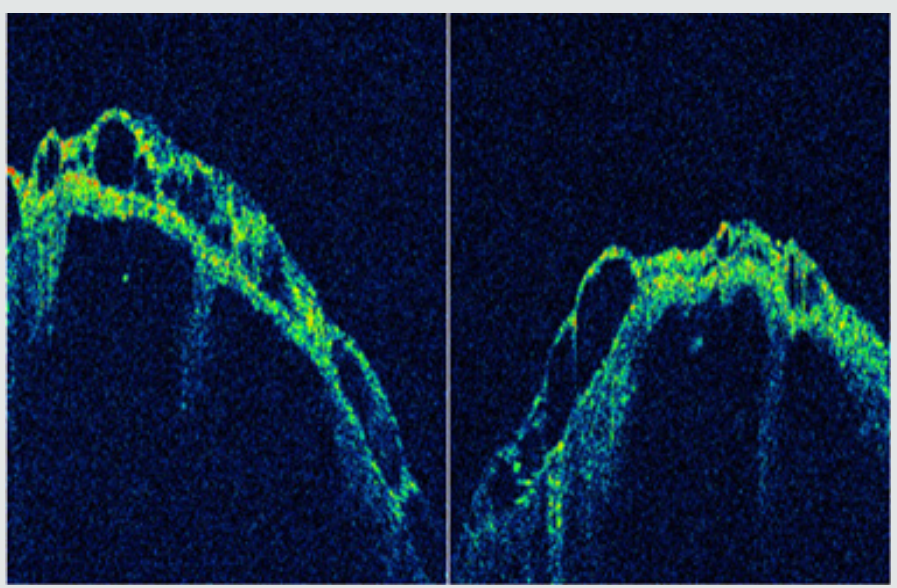

Figure 4: Anterior segment OCT of the left eye showing massive microcystic corneal edema and overlying epithelial bullae.

A 16-year-old Indian male presented with photophobia and a central loss of vision in his left eye 3 days ago. He has a positive history of Down syndrome associated with Keratoconus. The best corrected visual acuity (BCVA) for far and near was unable to be tested because of his sever mental retardation. Extraocular muscle movements were normal. Gross inspection of the face and lids was negative for ecchymosis, edema or asymmetry. Anterior segment evaluation on slit lamp biomicroscope revealed that the eyelids 
and lashes were clear without evidence of inflammation. The right cornea was clear, and the left cornea showed marked edema and central conical protrusion. The bulbar and palpebral conjunctivae were clear without injection, chemosis, melanosis, papillae or follicles. The Right eye showed Cerulean cataract; however, we could not assess the left eye due to marked edema. Color photography on the left eye detected corneal edema, bullae and conical protrusion (Figure 1). Pentacam of the right eye showed an early stage of Keratoconus (Figure 2) and of the left eye revealed marked increase in the corneal thickness (Figure 3). Anterior Chamber OCT Scan of the left eye revealed bullae edema but tear in DM was unable to photograph because patient was uncooperative during the investigation (Figure 4). Conservative treatment includes sodium chloride, cyclopentolate eye drops, combination of brimonidine and timolol with an artificial lubricant eye drops for symptomatic relief. A contact lens could not be used because the patient was uncooperative. Intracameral C3F8 gas injection was recommended to speed the recovery if there was no improvement with the medical treatment.

\section{Prognosis}

Gradual improvement in 4 weeks but extensive residual scarring and corneal irregularities were the cause of reduced visual acuity. Penetrating Keratoplasty (PK) was discussed but it was difficult because of low economic status and his mental retardation.

\section{Discussion}

Keratoconus is characterized by progressive thinning and protrusion of the cornea. Thus, Patients suffer from irregular astigmatism and impairment in visual function [4]. Acute corneal hydrops is caused by tear in Descemet's membrane which allows influx of fluid from the aqueous humor to the corneal stroma leading to acute corneal edema and formation of cystic spaces. This edema remains for few weeks and rapid management is encouraged because it could prevent the formation of a fistula and leakage from the aqueous humor [5-6]. The edema tends to resolve with extensive scarring. Keratoconus is one of eye features of Down Syndrome. Eye rubbing is one of the underestimated risk factors for development of acute hydrops in Keratoconus, especially with Down's syndrome patients [7-9]. Conservative treatment includes hypertonic saline, lubricants, cyclopentolate and anti-glaucoma eye drops are used till spontaneous resolutions occurs [10]. Intracameral Air/Gas injections showed remarkable results by reattaching the Descemet membrane back to the corneal stroma. The Gas blocks fluid from $\mathrm{AC}$ to the cornea results in reduced corneal swelling and accelerated healing process. PK is usually the treatment of choice in ectatic patients who previously developed hydrops, studies have been inconclusive regarding differences in graft survival between grafts with prior episodes of hydrops and grafts without prior hydrops [11].

\section{Conclusion}

Acute corneal hydrops should be considered one of theimportant eye features for the diagnosis of Down syndrome. Patients and their family should be warned of the eye rubbing. Treatment could be conservative or interventional, including intracameral injections and Penetrating Keratoplasty which is very challenging in mentally retarded patients. New treatments such as compressive sutures and tissue adhesive should be considered. We believe that there is no establishing approach to deal with a challenging problem like acute hydrops in down's syndrome patient with severe mental retardation. Those patients can not co-operate during examination and intervention therapy. They have behavioral disorders, hearing problems in addition to decreased cognitive functions. Educational programs for patients, family and doctors are necessary. Moreover, routine screening could be the most important method to discover the disorders earlier and may help prevent future complications.

\section{References}

1. Rabinowitz Y S (1998) Survey of Ophthalmology. Keratoconus 42(4): 297-319.

2. Fan Gaskin JC, Patel DV, McGhee CN (2014) Acute corneal hydrops in keratoconus - new perspectives. Am J Ophthalmol 157(5): 921-928.

3. Maharana PK, Sharma N, Vajpayee RB (2013) Acute corneal hydrops in keratoconus. Indian J Ophthalmol 61(8): 461-464.

4. Vazirani J, Basu S (2013) Keratoconus: current perspectives. Clin Ophthalmol 7: 2019-2030.

5. Grewal S, Laibson PR, Cohen EJ (1999) Acute hydrops in the corneal ectasias: associated factors and outcomes. Trans Am Ophthalmol Soc 97: 187-203.

6. Rubsamen PE, McLeish WM (1991) Keratoconus with acute hydrops and perforation. Brief case report. Cornea 10: 83-84.

7. Ozcan AA, Ersoz TR (2007) Severe acute corneal hydrops in a patient with Down syndrome and persistent eye rubbing. Ann Ophthalmol (Skokie) 39: 158-160.

8. McMonnies CW (2009) Mechanisms of rubbing-related corneal trauma in keratoconus. Cornea 28(6): 607-615.

9. Ioannidis AS, Speedwell L, Nischal KK (2005) Unilateral keratoconus in a child with chronic and persistent eye rubbing. Am J Ophthalmol 139(2): 356-357.

10. Tuift SJ, Gregory WM, Buckley RJ (1994) Acute corneal hydrops in keratoconus. Ophthalmology 101(10): 1738-1744.

11. Akova YA, Dabil H, Kavalcioglu O, Duman S (2000) Clinical features and keratoplasty results in keratoconus complicated by acute hydrops. Ocul Immunol Inflamm 8(2): 101-109. 
This work is licensed under Creative Commons Attribution 4.0 License

To Submit Your Article Click Here:

Submit Article

DOI: $10.32474 /$ TOOAI.2019.02.000143

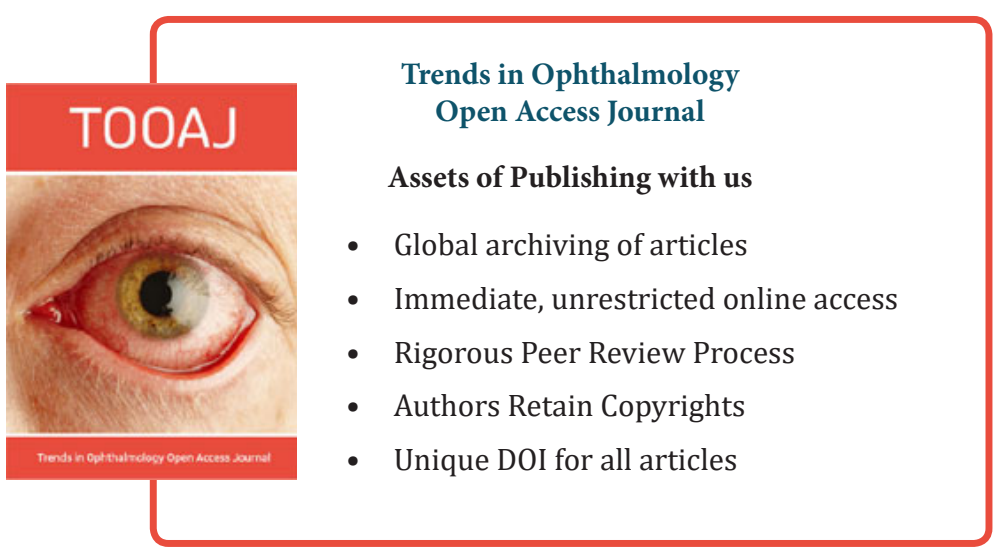

